micelle, containing enclosed oil, described by Lawrence $^{4}$; and since it contains enclosed water, and is tolerated by (though not attracted by) an oily dispersion medium, it may conveniently be termed the 'oleopathic hydro-micelle'.

Metallurgical Laboratories,

University, Cambridge.

Department of Colloid Science, University, Cambridge.

${ }^{1}$ SchuIman and Hughes, Biochem. $J$., 29, 1243 (1935). Schulman and Rideal, Proc. Roy. Soc., B, 122, 29, 46 (1937). Schulman and Stenhagen, Proc. Roy. 'Soc., B, 126, 356 (1938). Marsden and Schulman, Trans. Faraday Soc., 34, 748 (1939)., Lawrence, Trans. Faraday Soc., 35, 702 (1939).

'Reichenberg, unpublished work.

${ }^{3}$ Schulman and Cockbain, Trans. Faraday Soc., 36, 651, 661 (1940) 'Lawrence, Trans. Faraday Soc., 33, 325 (1937).

\section{Dual Decay of Potassium}

IT has been known for some time that the $\beta$-ray activity shown by potassium is due entirely to the isotope ${ }^{40} \mathrm{~K}$, which thereby transforms to ${ }^{40} \mathrm{Ca}$. One might expect that ${ }^{40} \mathrm{~K}$ would exhibit a dual decay, transforming to ${ }^{40} \mathrm{~A}$ either by emission of a positron or by the electron-capture process. Attempts have been made at various times to detect positrons in the radiation from potassium but without success. These experiments, however, would not have detected the electron-capture process. In 1937, von Weizsäcker pointed out that considerations of the abundance of the chemical elements suggested that the isotope ${ }^{40} \mathrm{~K}$ may in fact transform by a capture process to ${ }^{40} \mathrm{~A}$. The argument was as follows. The abundance of the chemical elements varies on the whole smoothly with atomic number, and the inert gases as a class show the same variation, except that they are rarer by several powers of 10 than their neighbouring elements. Argon is a striking exception, for it is about 1,000 times more abundant than would be expected from the general rule. The excess is due entirely to ${ }^{40} \mathrm{~A}$, and argon would obey the rule if the ${ }^{40} \mathrm{~A}$ atoms could be attributed entirely to the transformation of ${ }^{40} \mathrm{~K}$.

Some time ago, at the suggestion of the late Dr. Harold Walke, we examined the radiation from potassium for evidence of the $K$-capture process, by placing a tray containing a layer of potassium salt in a large expansion chamber.

When an electron of the $K$-shell is captured by the nucleus, its place must be taken almost immediately by an electron from an outer shell, with the emission of a quantum of characteristic $K$-radiation. In the present case, the quantum will be emitted by an argon nucleus and its energy will be rather less than 4,000 electron volts. Practically all the radiation escaping from the layer of potassium salt into the air of the chamber will be absorbed within a few centimetres of the source. The absorption of each quantum gives rise to a photo-electron of about $0.5 \mathrm{~mm}$. range, appearing in the cloud chamber as a large spot, easy to distinguish from any background cloud which may be present. The expansion chamber thus affords a sensitive method for the detection of the capture process, provided that the $\beta$-rays from the source of potassium salt are bent away by a strong magnetic field so as not to interfere with the observation of the spots.

In the course of the experiments 172 photographs

were taken, some using layers of potassium chloride and some potassium nitrate; on these, twenty-eight large spots were observed, nearly all within $3-4 \mathrm{~cm}$. of the source. There is good reason to believe that these spots are not a background effect of the chamber, for 117 photographs, taken under similar conditions except that layers of sodium salts were used, showed only two spots; while fifty photographs with no source gave no spots. The results show therefore that potassium emits an X-radiation of about the expected quantum energy, but it must be borne in mind that this radiation might arise from other processes than the $K$-capture process ; as, for example, by the ionization of the $K$-level of potassium by the $\beta$-radiation. Calculation indicates, however, that these other effects are far too weak to account for the observations, and there seems to be little doubt that the $K$-radiation arises from the electron capture process

$$
{ }^{40} \mathrm{~K} \rightarrow{ }^{40} \mathrm{~A} \text {. }
$$

An estimate has been made of the probability of this process relative to the known $\beta$-transformation to ${ }^{40} \mathrm{Ca}$. Owing to the great absorption of the $K$ radiation in the layer of salt and the uncertainty of the absorption coefficient, the calculations are very rough. They lead to the conclusion that the branch. ing ratio is about three or four disintegrations by electron capture to one by electron emission, whereas von Weizsäcker estimated the ratio to be 1 to 3 . As both estimates are liable to large errors, the discrepancy cannot be regarded as serious.

Recalculating the half-value period of ${ }^{40} \mathrm{~K}$ to take account of this alternative mode of decay, we arrive at a value of about $4 \times 10^{8}$ years, not an unreasonable figure.

It would be interesting to be able to show that old potassium minerals, for example, granites or potassium feldspars, contain argon in amount corresponding to their age and potassium content. There exists already evidence of the presence of argon in granites ${ }^{1}$ but its significance is not clear, for it is difficult to exclude completely the possibility of atmospheric contamination. The outbreak of war and the pressure of other interests prevented us from pursuing this aspect of the problem, and there seems little prospect of our being able to take it up for some time. For this and other reasons we make known our results, although not as complete as we would wish, without further delay.

F. C. Thompson. S. Rowlands.

George Holt Physics Laboratory,

University of Liverpool. June 21.

1 cf. Rayleigh, NATURE, 141, 410 and 970 (1938); and Shepherd, Amer. J. Sci., 35A, 311 (1938).

\section{Nature of Tetraploidy in Cultivated European Potatoes}

IT must be unusual to find that the essential details of the genetic behaviour of an economic crop of such standing as the potato can still offer fertile grounds for controversy. Yet such is indeed the case.

The relevant facts of the cytogenetic situation are briefly these. The family Solanacex, to which the potato belongs, appears to be characteristically poly. ploid on the basic number 12, $2 n=24$ being the lowest chromosome number actually found. The European 